\title{
Targeting Chemoprevention of Colorectal Cancer to Those Who Are Likely to Respond
}

\author{
Reinhold W. Stockbrugger
}

Published online: 9 January 2010

(C) The Author(s) 2010. This article is published with open access at Springerlink.com

\begin{abstract}
In the past four decades, chemoprevention of colorectal cancer (CRC) has been the subject of many epidemiologic and intervention trials of naturally occurring or pharmacologic agents. Recently, the positioning of cyclooxygenase 2 inhibitors as a viable option in this context was a major breakthrough; however, it was hampered by adverse cardiovascular events. This review questions whether chemopreventive measures for $\mathrm{CRC}$ are ready to be used in mass or individual applications, standing alone or in combination with other CRCpreventive measures. It also discusses steps that may be undertaken to explore this field further.
\end{abstract}

Keywords Chemoprevention - Colorectal cancer .

COX-2 antagonists · Primary and secondary prevention.

\section{Introduction}

The task of this overview can be resolved easily or not at all. If we want to understand chemoprevention of colorectal cancer (CRC) as either the administration of favorable chemical agents (natural or synthetic) or the exclusion of adversely acting compounds, we would be able to indicate an effect on nearly every human subject, even if the individual gain is very small. On the contrary, if we aim at a significant, well-defined, and tangible CRC-preventive effect in individual patients with normal or high risk for the occurrence of precancerous or cancerous colorectal

R. W. Stockbrugger $(\square)$

Department of Internal Medicine,

Maastricht University Medical Center,

Debyelaan 25,

AZ 6202 Maastricht, Netherlands

e-mail: reinholdstockbrugger@gmail.com lesions, the outcome may be poor or at least very unreliable. However, for both options there are ways to develop and-together with nonchemical interventionprovide quite effective CRC prevention now. We can consider primary, secondary, and tertiary prevention in this context.

\section{Should We Aim at a Mass Effect?}

If we want a mass effect of CRC prevention, we would, for example, consider preventive actions in populations with a high incidence and considerable lifetime risk of developing CRC (eg, about $6 \%$ in the Western world) [1]. Some "chemical" risk factors or risk situations are clearly defined in this context, including the nutritional causes of the metabolic syndrome, which is associated with a risk of $\mathrm{CRC}$ as well as many other malignancies [2]. Let us be clear: if the overnutrition of a large proportion of certain populations had been avoided some decades ago and if adequate dietary and lifestyle advice had been given at an early stage, Western medicine could have stopped the surge of CRC that is still occurring in several countries. If such actions had been combined with a reduction in tobacco use, we would have saved many lives and immense sums in health care expenses (including those necessary for forming armies of endoscopists, nurses, and other health care workers).

Fortunately, it is not too late for action: applying such measures to large uninformed and innocent populations, such as children and those in underdeveloped or developing countries who are repeating the mistakes of Western cultures, might at least partly reduce our feelings of professional failure and guilt. We must acknowledge that being a physician today involves much more 
than treating and potentially curing patients. Prevention in the widest sense is a "must," not only ethically but also economically.

Reversing the process of latent colorectal carcinogenesis in populations composed of both healthy- and unhealthy-living individuals by adding one or several defined chemical substances to their diet carries much promise. However, in the proportion of these populations with high lifestyle risks for precancerous or cancerous lesions, the critical time point for reversing carcinogenic mechanisms may already have passed, and intervention may give few or no results. Most intervention studies performed in these populations and using vitamins, fiber, and other agents showed either no or marginal effects. Whether adding synthetic and stronger CRC-preventive compounds can improve the prognosis of such groups with respect to CRC has, to my knowledge, not yet been studied and certainly cannot be excluded. Trials examining this question-either prospective or retrospectivewould necessitate clear definitions of healthy and unhealthy lifestyles and would require huge numbers of participants and/or lengthy study periods. Once studies are undertaken, they should include outcome parameters regarding not only CRC, but also other malignant or benign disorders.

\section{CRC Chemoprevention for High-Risk Patients}

Should we offer CRC chemoprevention to patients at very high risk? Of course we should. These are the patients who have undergone successful CRC treatment (tertiary prevention), those who have had adenomatous polyps removed (secondary prevention), and those at high risk because of accumulated risk factors-lifestyle, genetic, and hereditary-(primary prevention). Patients at high risk also include, in extreme cases, those with familial adenomatous polyposis, for whom there already is a US Food and Drug Administration-approved indication for the use of cyclooxygenase 2 (COX-2) inhibitors $[3 \cdot \bullet]$. In the risk/effect calculation, we would need agents with considerable proven effect and safety margins that could guarantee a significant health gain in small populations. These types of preventive interventions are similar to drug therapies for non-malignant and less severe disorders, for which we are prone to accept adverse effects to a certain degree if the net effect is convincing, such as in the use of aspirin to prevent cardiovascular fatalities [4]. However, there are experts who argue that "preventive actions" should never be harmful. The balance between the chances of benefit and harm in these situations should always be discussed with patients in a clear and straightforward way. Also, besides approval by the institutions responsible for drug indications, patients' informed consent must be obtained and documented. Such consent should be obtained only after patients have been offered a thorough explanation of the advantages and disadvantages of the current "gold standard" in CRC screening, that is, optical colonoscopy with the eventual endoscopic removal of precancerous and minor cancerous lesions.

\section{Colonoscopy: The Mainstay of CRC Prevention}

Many methods are currently available for detecting premalignant and malignant lesions of the colon, and new ones are on the way [5]. These methods range from optical techniques (x-ray, CT, MRI, manual high-resolution colonoscopy, and semiautomated colonoscopy) to biochemical, immunologic, and genetic tests of feces or blood. None of the latter (non-imaging) methods is accurate enough yet to detect colorectal neoplasia within the necessary range of sensitivity and specificity. In the imaging field, trials and discussions are ongoing to determine whether virtual colonoscopy with CT (CT colonography) can be used to select participants for screening with optical colonoscopy with the possibility of removing any "real" lesions discovered [6]. The semiautomated endoscopic approaches are still under study for the same purpose. However, one must realize that even with the best available techniques, total optical colonoscopy with biopsies and/or other methods of removal (snare polypectomy, mucosectomy) remains a necessity for solving the problem, notwithstanding the discomfort and adverse effects this method may have, even in the hands of the best-trained endoscopists. It is estimated that about $40 \%$ of screening CT colonographies result in a positive or doubtful finding requiring optical colonoscopy [7].

Unfortunately, campaigns for CRC screening pay too little attention to the fact that the most frequently used nonimaging screening method - the fecal occult blood test (FOBT), as well as its advanced immunologic version (iFOBT) - has a sensitivity for invasive CRCs of $70 \%$ or less, and that the sensitivity is much lower for precancerous lesions, otherwise known as advanced neoplasia $(<30 \%)$ [8]. Comparing these relatively low figures with the estimated (unfortunately, not yet prospectively proven) possibility of reducing CRC mortality by more than $80 \%$ with primary colonoscopy and lesion removal, with an examination every 10 years after the age of 50 [9], should lead to the conclusion that - in CRC screening programsoptical colonoscopy should always be one of the options provided to screenees, even if less invasive methods have been selected for the majority of the population for economic or organizational reasons. 


\section{Chemoprevention and Colonoscopy}

Having clarified that none of the presently available methods for CRC prevention can reach a result comparable with optical detection and instrumental removal of colorectal neoplasia, the following questions must be answered:

1. Is a combination of chemoprevention and colonoscopy advisable? If so, in which design?

2. Are there suitable compounds for the chemoprevention part of such a combination?

3. How do we characterize or identify people who would benefit from a combination strategy?

\section{Combination Colonoscopy and Chemoprevention}

It is obvious that - for now and in the near future- neither general nor individual chemoprevention with lifestylerelated and/or pharmacologic chemoprevention can replace optical examination of the colon if the objective is early detection or prevention of CRC. The recently published forerunner trials performed with $\mathrm{COX}-2$ antagonists have clearly indicated that a partial reduction in recurrent colonic adenomas (by about $30 \%$ to $40 \%$ ) is possible for at least 3 years $[10 \bullet \cdot$, but at the cost of considerable cardiovascular adverse effects [11]. Even if this strategy had been approved by national or international health authorities, the trials performed did not give a secure clue about individual screenee characteristics that would predict a potential benefit from the pharmacologic intervention, precluding prospective selection for such a combined strategy. Compared with the very precise pharmacologic studies testing the value of COX-2 inhibitors, an assumption of the effect of lifestyle-related chemoprevention on individual screenees must remain even more approximate or-clearly spoken-impossible. Accepting these facts, there is still a need to identify safer and more effective chemoprevention methods and to retest them in trials using colonoscopic assessment of the outcome, as was done in the aforementioned experiments with COX-2 inhibitors.

\section{Candidates for CRC Chemoprevention in the Near Future}

Based on the available knowledge from human and animal studies, there is a limited number of agents available for further testing, either alone or in different combinations $[3 \bullet \bullet$. They will be based mostly on experience gained from controlled epidemiologic and/or intervention studies or from animal models, and might combine reduced doses of known pharmacologic agents (eg, aspirin or COX-2 inhibitors) with other promising compounds, such as selenium or curcumin $[3 \bullet \bullet, 12]$. However, before controlled human studies can be performed, it will take more than single short-term animal experiments to avoid the immense investment of human and economic resources necessary to develop more effective and safer versions of CRC chemoprevention. In this context, using previously adopted protocols and procedures, such as those of the PreSAP (Prevention of Colorectal Sporadic Adenomatous Polyps) and APC (Adenoma Prevention With Celecoxib) trials $[10 \bullet, 11]$, would improve comparability of outcomes.

Identifying the Screenees to be Targeted for a Combined Colonoscopy/Chemoprevention Strategy

On the basis of existing scientific evidence, it is impossible to identify the individuals or populations that, with any certainty, could be directed to a combined CRC colonoscopy/chemoprevention strategy. Identification of target group characteristics for a similar approach should be part of any future testing of chemopreventive agents and should include biological as well as psychological and social factors with a potential impact. Also, biological material from participants in such trials should be obtained and preserved (ie, bio-banked), because possible "markers" for the susceptibility, tolerability, and economic utility of future chemopreventive options may potentially be investigated with the help of such preserved material.

\section{In the Meantime}

It is frustrating not to have an answer to the question of who would benefit from CRC chemoprevention without risking insufficient efficacy and/or hazardous side effects. At present, attempts in this direction might result in professionalincluding legal - consequences. In considering any patient for chemopreventive alternatives to the accepted ("conventional") CRC screening methods, one must clearly account for the statistical validity of such measures, albeit with an explanation of evidence lacking for individual applicability. Also, in light of the present gold standard for CRC prevention, one should consider how to proceed with a patient at high risk for CRC who refuses a screening colonoscopy (or has relative or absolute contraindications for it) in the setting of clinical indications or a high suspicion of CRC based on any noncolonoscopic screening method. In this difficult situation, many patients and their medical advisers would reconsider and choose the option of colonoscopy, asking for the best available standards of preparation, technique, and follow-up.

\section{Conclusions}

The presently available lifestyle-related or chemopreventive options against CRC still have low or moderate efficacy 
compared with the current gold standard of optical colonoscopy and lesion removal. The most promising substances for chemoprevention-COX-2 inhibitors-were not approved for CRC chemoprevention because of adverse cardiovascular events. Future therapies should be tested with a comparable accuracy to avoid failure and/or negative side effects in persons and populations subjected to them. In the meantime, the quality of colonoscopy-based screening and follow-up procedures should be improved and adequate focused information regarding the population considered for CRC prevention should be provided to prevent further delays in the fight against this potentially avoidable malignancy.

Disclosure No potential conflict of interest relevant to this article was reported.

Open Access This article is distributed under the terms of the Creative Commons Attribution Noncommercial License which permits any noncommercial use, distribution, and reproduction in any medium, provided the original author(s) and source are credited.

\section{References}

Papers of particular interest, published recently, have been highlighted as:

- Of importance

- Of major importance

1. Vogelaar I, van Ballegooijen M, Schrag D, et al.: How much can current interventions reduce colorectal cancer mortality in the U.S.? Cancer 2006, 107:1624-1633.
2. Anderson JC, Messina CR, Dakhllahlah F, et al.: Body mass index, a marker for significant colorectal neoplasia in a screening population. J Clin Gastroenterol 2007, 41:285-290.

3. • Das D, Arber N, Jankowski JA: Chemoprevention of colorectal cancer. Digestion 2007, 76:51-67. This is an excellent review of existing and future options for chemoprevention of CRC.

4. Dutch TIA Trial Study Group: A comparison of two doses of aspirin (30 mg vs $283 \mathrm{mg}$ a day) in patients after a transient ischemic attack or minor ischemic stroke. N Engl J Med 1991, 325:1261-1266.

5. Winawer SJ, Zauber AG, Fletcher RH, et al.: Guidelines for colonoscopy surveillance after polypectomy: a consensus update by the US Multi-Society Task Force on Colorectal Cancer and the American Cancer Society. Gastroenterology 2006, 2006:18721885.

6. Rockey DC, Barish M, Brill JV, et al.: Standards for gastroenterologists for performing and interpreting diagnostic computed tomographic colonography. Gastroenterology 2007, 133:10051024.

7. Kim DH, Pickhardt PJ, Taylor AJ, et al.: Colonography versus colonoscopy for the detection of advanced neoplasia. N Engl J Med 2007, 357:1403-1412.

8. Morikawa T, Kato J, Yamaji Y, et al.: A comparison of the immunochemical fecal occult blood test and total colonoscopy in the asymptomatic population. Gastroenterology 2005, 129:422428.

9. Winawer SJ, Zauber AG, Ho MN, et al.: Prevention of colorectal cancer by colonoscopic polypectomy. N Engl J Med 1993, 329:1977-1981.

10. • Arber N, Eagle CJ, Spicak J, et al.: Celecoxib for the prevention of colorectal adenomatous polyps. N Engl J Med 2006, 355:885895. This landmark study provides a model for testing future chemopreventive actions against CRC.

11. Solomon SD, McMurray JJ, Pfeffer MA, et al.: Cardiovascular risk associated with celecoxib in a clinical trial for colorectal adenoma prevention. N Engl J Med 2005, 352:1071-1080.

12. Shpitz B, Giladi N, Sagiv E, et al.: Celecoxib and curcumin additively inhibit the growth of colorectal cancer in a rat model. Digestion 2006, 74:140-144. 